\title{
SOME CONVERGENCE THEOREMS FOR OPERATOR SEQUENCES
}

\author{
HEYBETKULU MUSTAFAYEV
}

\begin{abstract}
Let $A, T$ and $B$ be bounded linear operators on a Banach space. This paper is concerned mainly with finding some necessary and sufficient conditions for convergence in operator norm of the sequences $\left\{A^{n} T B^{n}\right\}$ and $\left\{\frac{1}{n} \sum_{i=0}^{n-1} A^{i} T B^{i}\right\}$. These results are applied to the Toeplitz, composition and model operators. Some related problems are also discussed.
\end{abstract}

\section{INTRODUCTION}

Throughout this paper, $H$ will denote a complex separable infinite dimensional Hilbert space and $B(H)$, the algebra of all bounded linear operators on $H$. The ideal of all compact operators on $H$ will be denoted by $K(H)$. The quotient algebra $B(H) / K(H)$ is a $C^{*}$-algebra and called the Calkin algebra. As usual, $H^{2}$ will denote the classical Hardy space on the open unit disk $\mathbb{D}:=\{z \in \mathbb{C}:|z|<1\}$. By $H^{\infty}$ we will denote the space of all bounded analytic functions on $\mathbb{D}$.

Let $\mathbb{T}:=\partial \mathbb{D}$ be the unit circle and let $m$ be the normalized Lebesgue measure on $\mathbb{T}$. Recall that for a given symbol $\varphi \in L^{\infty}:=L^{\infty}(\mathbb{T}, m)$, the Toeplitz operator $T_{\varphi}$ on $H^{2}$ is defined by

$$
T_{\varphi} f=P_{+}(\varphi f), \quad f \in H^{2},
$$

where $P_{+}$is the orthogonal projection from $L^{2}(\mathbb{T}, m)$ onto $H^{2}$. Let

$$
S f(z)=z f(z)
$$

be the unilateral shift operator on $H^{2}$. According to a theorem of Brown and Halmos [3], $T \in B\left(H^{2}\right)$ is a Toeplitz operator if and only if

$$
S^{*} T S=T \text {. }
$$

Barria and Halmos [1] examined the so-called strongly asymptotically Toeplitz operators $T$ on $H^{2}$ for which the sequence $\left\{S^{* n} T S^{n}\right\}$ converges strongly. This class includes the Hankel algebra, the operator norm-closed algebra generated by all Toeplitz and Hankel operators together [1.

An operator $T \in B\left(H^{2}\right)$ is said to be uniformly asymptotically Toeplitz if the sequence $\left\{S^{* n} T S^{n}\right\}$ converges in the uniform operator topology. This class of operators is closed in operator norm and under adjoints. It contains both Toeplitz

2010 Mathematics Subject Classification. 47A11, 47A35, 47B07; 47B35.

Key words and phrases. Hilbert space, Banach space, compact operator, Toeplitz operator, composition operator, model operator, local spectrum, operator sequence, operator average, convergence. 
operators and the compact ones. Feintuch [8] proved that an operator $T \in B\left(H^{2}\right)$ is uniformly asymptotically Toeplitz if and only if it has the decomposition

$$
T=T_{0}+K
$$

where $T_{0}$ is a Toeplitz operator, that is, $S^{*} T_{0} S=T_{0}$ and $K$ is a compact operator.

Recall that each holomorphic function $\phi: \mathbb{D} \rightarrow \mathbb{D}$ induces a bounded linear composition operator $C_{\phi}$ on $H^{2}$ by $C_{\phi} f=f \circ \phi$ (for instance, see [16, Ch.5]). The only composition operator, which is also Toeplitz, is the identity operator 20]. Using Feintuch's result, Nazarov and Shapiro [20, Theorem 1.1] proved that a composition operator on $H^{2}$ is uniformly asymptotically Toeplitz if and only if it is either compact or the identity operator.

Let $B(X)$ be the algebra of all bounded linear operators on a complex Banach space $X$ and let $A, T$ and $B$ be in $B(X)$. The main purpose of this paper is to find necessary and sufficient conditions for convergence in operator norm of the sequences $\left\{A^{n} T B^{n}\right\}$ and $\left\{\frac{1}{n} \sum_{i=0}^{n-1} A^{i} T B^{i}\right\}$.

\section{The SEQUEnCE $\left\{A^{n} T B^{n}\right\}$}

In this section, we give some results concerning convergence in operator norm of the sequence $\left\{A^{n} T B^{n}\right\}$ for Hilbert space operators.

Recall that an operator $T \in B(H)$ is said to be essentially isometric (resp. essentially unitary) if $I-T^{*} T \in K(H)$ (resp. $I-T^{*} T \in K(H)$ and $I-T T^{*} \in$ $K(H))$.

We have the following:

Theorem 2.1. Let $A$ and $B^{*}$ be two essentially isometric operators on $H$ such that $\left\|A^{n} x\right\| \rightarrow 0$ and $\left\|B^{* n} x\right\| \rightarrow 0$, for all $x \in H$. If $T \in B(H)$, then the sequence $\left\{A^{n} T B^{n}\right\}$ converges in operator norm if and only if we have the decomposition

$$
T=T_{0}+K
$$

where $A T_{0} B=T_{0}$ and $K \in K(H)$.

For the proof, we need some preliminary results.

Let $H_{0}$ be the linear space of all weakly null sequences $\left\{x_{n}\right\}$ in $H$. Let us define a semi-inner product in $H_{0}$ by

$$
\left\langle\left\{x_{n}\right\},\left\{y_{n}\right\}\right\rangle=\text { l.i.m. }{ }_{n}\left\langle x_{n}, y_{n}\right\rangle,
$$

where l.i.m. is a fixed Banach limit. If

$$
E:=\left\{\left\{x_{n}\right\} \in H_{0}: \text { l.i.m.n }\left\|x_{n}\right\|^{2}=0\right\},
$$

then $H_{0} / E$ becomes a pre-Hilbert space with respect to the inner product defined by

$$
\left\langle\left\{x_{n}\right\}+E, \quad\left\{y_{n}\right\}+E\right\rangle=\text { l.i.m.n }\left\langle\left\{x_{n}\right\},\left\{y_{n}\right\}\right\rangle .
$$

Let $\widehat{H}$ be the Hilbert space defined by the completion of $H_{0} / E$ with respect to the induced norm

$$
\left\|\left\{x_{n}\right\}+E\right\|=\left(\text { l.i.m.n }\left\|x_{n}\right\|^{2}\right)^{\frac{1}{2}} .
$$

Now, for a given $T \in B(H)$ we define an operator $\widehat{T}$ on $H_{0} / E$ by

$$
\widehat{T}:\left\{x_{n}\right\}+E \mapsto\left\{T x_{n}\right\}+E .
$$


Consequently, we can write

$$
\begin{aligned}
\left\|\widehat{T}\left(\left\{x_{n}\right\}+E\right)\right\| & =\left(\text { l.i.m.n }\left\|T x_{n}\right\|^{2}\right)^{\frac{1}{2}} \\
& \leq\|T\|\left(\text { l.i.m. } \cdot\left\|x_{n}\right\|^{2}\right)^{\frac{1}{2}} \\
& =\|T\|\left\|\left\{x_{n}\right\}+E\right\| .
\end{aligned}
$$

Since $H_{0} / E$ is dense in $\widehat{H}$, the operator $\widehat{T}$ can be extended to the whole $\widehat{H}$ which we also denote by $\widehat{T}$. Clearly, $\|\widehat{T}\| \leq\|T\|$. The operator $\widehat{T}$ will be called limit operator associated with $T$.

Proposition 2.2. If $\widehat{T}$ is the limit operator associated with $T \in B(H)$, then:

(a) The map $T \mapsto \widehat{T}$ is a linear contractive $*$-homomorphism.

(b) $T$ is a compact operator if and only if $\widehat{T}=0$.

(c) $T$ is an essentially isometry (resp. essentially unitary) if and only if $\widehat{T}$ is an isometry (resp. unitary).

(d) For an arbitrary $T \in B(H)$, we have $\|\widehat{T}\|=\|T+K(H)\|$.

Proof. Proofs of the assertions (a), (b) and (c) are omitted, since they are clear. Let us prove (d). Let $\widehat{K}$ be the limit operator associated with $K \in K(H)$. Since $\widehat{K}=0$, we get

$$
\|\widehat{T}\|=\|\widehat{T}+\widehat{K}\| \leq\|T+K\|, \quad \forall K \in K(H) .
$$

This implies $\|\widehat{T}\| \leq\|T+K(H)\|$. For the reverse inequality, recall [2, p.94] that

$$
\|T+K(H)\|=\sup \left\{\varlimsup_{n \rightarrow \infty}\left\|T x_{n}\right\|:\left\|x_{n}\right\|=1, \forall n \in \mathbb{N} \text { and } x_{n} \rightarrow 0 \text { weakly }\right\} .
$$

Therefore, for a given $\varepsilon>0$ there exists a sequence $\left\{x_{n}\right\}$ in $H$ such that $\left\|x_{n}\right\|=1$ $(\forall n \in \mathbb{N}), x_{n} \rightarrow 0$ weakly and

$$
\varlimsup_{n \rightarrow \infty}\left\|T x_{n}\right\| \geq\|T+K(H)\|-\varepsilon .
$$

Consequently, there exists a subsequence $\left\{x_{n_{k}}\right\}$ of $\left\{x_{n}\right\}$ such that

$$
\lim _{k \rightarrow \infty}\left\|T x_{n_{k}}\right\| \geq\|T+K(H)\|-\varepsilon .
$$

On the other hand,

$$
\|\widehat{T}\|=\sup \left\{\left(\text { l.i.m.n }_{\cdot n}\left\|T x_{n}\right\|^{2}\right)^{\frac{1}{2}}: \text { l.i.m.n }\left\|x_{n}\right\|^{2}=1 \text { and } x_{n} \rightarrow 0 \text { weakly }\right\} .
$$

As l.i.m. \|\|$x_{n_{k}} \|^{2}=1$ and $x_{n_{k}} \rightarrow 0(k \rightarrow \infty)$ weakly, by the preceding identity we get

$$
\|\widehat{T}\| \geq \lim _{k \rightarrow \infty}\left\|T x_{n_{k}}\right\| \geq\|T+K(H)\|-\varepsilon .
$$

Since $\varepsilon$ is arbitrary, we have $\|\widehat{T}\| \geq\|T+K(H)\|$, as required.

Lemma 2.3. (a) Let $A, B \in B(H)$ and assume that $\left\|A^{n} x\right\| \rightarrow 0$ and $\left\|B^{* n} x\right\| \rightarrow 0$, for all $x \in H$. Then, for an arbitrary $K \in K(H)$, we have

$$
\lim _{n \rightarrow \infty}\left\|A^{n} K B^{n}\right\|=0 .
$$


(b) If $A$ and $B^{*}$ are essentially isometric operators and

$$
\lim _{n \rightarrow \infty}\left\|A^{n} T B^{n}\right\|=0,
$$

then $T$ is a compact operator.

Proof. (a) For an arbitrary $x, y \in H$, let $x \otimes y$ be the rank one operator on $H$;

$$
x \otimes y: z \mapsto\langle z, y\rangle x, \quad z \in H .
$$

Since finite rank operators are dense (in operator norm) in $K(H)$, we may assume that $K$ is a finite rank operator, say,

$$
K=\sum_{i=1}^{N} x_{i} \otimes y_{i}
$$

where $x_{i}, y_{i} \in H(i=1, \ldots, N)$. Consequently, we can write

$$
\left\|A^{n} K B^{n}\right\|=\left\|\sum_{i=1}^{N} A^{n} x_{i} \otimes B^{* n} y_{i}\right\| \leq \sum_{i=1}^{N}\left\|A^{n} x_{i}\right\|\left\|B^{* n} y_{i}\right\| \rightarrow 0 \quad(n \rightarrow \infty) .
$$

(b) Let $\widehat{A}, \widehat{T}$ and $\widehat{B}$ be the limit operators associated with $A, T$ and $B$, respectively. By Proposition 2.2, $\widehat{A}$ and $\widehat{B}^{*}$ are isometries. Since the map $T \mapsto \widehat{T}$ is a contractive homomorphism, for an arbitrary $n \in \mathbb{N}$ we get

$$
\|\widehat{T}\|=\left\|\widehat{A}^{n} \widehat{T} \widehat{B}^{n}\right\| \leq\left\|A^{n} T B^{n}\right\| \rightarrow 0 .
$$

Hence $\widehat{T}=0$. By Proposition 2.2, $T$ is a compact operator.

We are now in a position to prove Theorem 2.1.

Proof of Theorem 2.1. If $T=T_{0}+K$, where $A T_{0} B=T_{0}$ and $K \in K(H)$, then

$$
A^{n} T B^{n}=T_{0}+A^{n} K B^{n}, \quad \forall n \in \mathbb{N} .
$$

By Lemma 2.3, $\left\|A^{n} K B^{n}\right\| \rightarrow 0$ and therefore $\left\|A^{n} T B^{n}-T_{0}\right\| \rightarrow 0$. Now, assume that there exists $T_{0} \in B(H)$ such that $\left\|A^{n} T B^{n}-T_{0}\right\| \rightarrow 0$. Since

$$
\left\|A^{n+1} T B^{n+1}-A T_{0} B\right\| \rightarrow 0,
$$

we have $A T_{0} B=T_{0}$ which implies $A^{n} T_{0} B^{n}=T_{0}$ for all $n \in \mathbb{N}$. Also, since

$$
\left\|A^{n}\left(T-T_{0}\right) B^{n}\right\| \rightarrow 0,
$$

by Lemma 2.3, $T-T_{0}$ is a compact operator. So we have $T=T_{0}+K$, where $K \in K(H)$.

As a consequence of Theorem 2.1 we have the following:

Corollary 2.4. Let $A \in B(H)$ and assume that $I-A A^{*} \in K(H)$ and $\left\|A^{* n} x\right\| \rightarrow 0$ for all $x \in H$. If $T \in B(H)$, then the sequence $\left\{A^{* n} T A^{n}\right\}$ converges in operator norm if and only if we have the decomposition $T=T_{0}+K$, where $A^{*} T_{0} A=T_{0}$ and $K \in K(H)$.

If $S$ is the unilateral shift on $H^{2}$, then the operator $I-S S^{*}$ is one dimensional and $\left\|S^{* n} f\right\| \rightarrow 0$ for all $f \in H^{2}$. By taking $A=S$ in Corollary 2.4, we obtain Feintuch's result mentioned above.

Let an arbitrary $\varphi, \psi \in L^{\infty}$ be given. As we have noted in the Introduction, $T_{\varphi} T_{\psi}$ is a strongly asymptotically Toeplitz operator, that is, $S^{* n} T_{\varphi} T_{\psi} S^{n} \rightarrow T_{\varphi \psi}$ strongly [1, Theorem 4]. From this and from Corollary 2.4 it follows that $T_{\varphi} T_{\psi}$ 
is a uniformly asymptotically Toeplitz operator if and only if $T_{\varphi} T_{\psi}$ is a compact perturbation of the Toeplitz operator $T_{\varphi \psi}$. Now, assume that one of the functions $\varphi, \psi$ is a trigonometric polynomial, say, $\psi=\sum_{-N}^{N} c_{k} e^{i k \theta}$. Then as

$$
T_{\psi}=\sum_{k=1}^{N} c_{-k} S^{* k}+\sum_{k=0}^{N} c_{k} S^{k}
$$

$S^{* n} T_{\varphi} S^{* k} S^{n}=S^{* k} T_{\varphi}(\forall n \geq k)$ and $S^{* n} T_{\varphi} S^{k} S^{n}=T_{\varphi} S^{k}(\forall k \geq 0)$, we have

$$
S^{* n} T_{\varphi} T_{\psi} S^{n}=\sum_{k=1}^{N} c_{-k} S^{* k} T_{\varphi}+\sum_{k=0}^{N} c_{k} T_{\varphi} S^{k}, \quad \forall n \geq N .
$$

If $\varphi=\sum_{-N}^{N} c_{k} e^{i k \theta}$, then as $S^{* n} S^{* k} T_{\psi} S^{n}=S^{* k} T_{\psi}(\forall k \geq 0)$ and $S^{* n} S^{k} T_{\psi} S^{n}=$ $T_{\psi} S^{k}(\forall n \geq k)$, we have

$$
S^{* n} T_{\varphi} T_{\psi} S^{n}=\sum_{k=1}^{N} c_{-k} S^{* k} T_{\psi}+\sum_{k=0}^{N} c_{k} T_{\psi} S^{k}, \quad \forall n \geq N .
$$

Therefore, if one of the functions $\varphi, \psi$ is continuous, then $T_{\varphi} T_{\psi}$ is a uniformly asymptotically Toeplitz operator. Further, if $\psi$ has the form $\psi=h+f$, where $h \in H^{\infty}$ and $f \in C(\mathbb{T})$, then as $T_{\varphi} T_{h}=T_{\varphi h}$ we get

$$
\begin{aligned}
S^{* n} T_{\varphi} T_{\psi} S^{n} & =S^{* n} T_{\varphi}\left(T_{h}+T_{f}\right) S^{n} \\
& =S^{* n} T_{\varphi h} S^{n}+S^{* n} T_{\varphi} T_{f} S^{n} \\
& =T_{\varphi h}+S^{* n} T_{\varphi} T_{f} S^{n} .
\end{aligned}
$$

It follows that $T_{\varphi} T_{\psi}$ is a uniformly asymptotically Toeplitz operator for all $\varphi \in L^{\infty}$ and $\psi \in H^{\infty}+C(\mathbb{T})$ (recall that the algebraic sum $H^{\infty}+C(\mathbb{T}$ ) is a uniformly closed subalgebra of $L^{\infty}$ and sometimes called a Douglas algebra). Consequently, $T_{\varphi} T_{\psi}$ is a compact perturbation of the Toeplitz operator $T_{\varphi \psi}$ for all $\varphi \in L^{\infty}$ and $\psi \in H^{\infty}+C(\mathbb{T})$. Similarly, we can see that if $\varphi$ has the form $\varphi=\bar{h}+f$, where $h \in H^{\infty}$ and $f \in C(\mathbb{T})$, then $T_{\varphi} T_{\psi}$ is a uniformly asymptotically Toeplitz operator.

Note that in Corollary 2.4, compactness condition of the operator $I-A A^{*}$ is essential. To see this, let $A=V$ be the Volterra integral operator on $H=L^{2}[0,1]$. Then, $I-V V^{*} \notin K(H)$ and as $\left\|V^{n}\right\| \rightarrow 0$, we have $\left\|V^{* n} x\right\| \rightarrow 0$ for all $x \in H$. Since $\left\|V^{* n} T V^{n}\right\| \rightarrow 0$ for all $T \in B(H)$, the equation $V^{*} T_{0} V=T_{0}$ has only zero solution. If the conclusion of Corollary 2.4 were true, we would get $B(H) \subseteq K(H)$, which is a contradiction.

Let $H^{2}(E)$ be the Hardy space of all analytic functions on $\mathbb{D}$ with values in a Hilbert space $E$. Let $A \in B(H)$ be a contraction, $E:=\overline{\left(I-A A^{*}\right) H}$ and assume that $\left\|A^{* n} x\right\| \rightarrow 0$ for all $x \in H$. By the Model Theorem of Nagy-Foiaş (see, [19, Ch.VI, Theorem 2.3] and 21]), $A$ is unitary equivalent to its model operator

$$
A_{\Theta} f:=P_{\mathcal{K}} S_{E} f, \quad f \in \mathcal{K},
$$

where $\mathcal{K}=H^{2}(E) \ominus \Theta H^{2}(F), F$ is a subspace of $E, \Theta$ is a bounded analytic function on $\mathbb{D}$ with values in $B(F, E)$, the space of all bounded linear operators from $F$ into $E(\Theta(\xi)$ is an isometry for almost all $\xi \in \mathbb{T}), P_{\mathcal{K}}$ is the orthogonal projection from $H^{2}(E)$ onto $\mathcal{K}$ and $S_{E}$ is the unilateral shift operator on $H^{2}(E)$. Notice also that $A_{\Theta}^{*}=S_{E}^{*} \mid \mathcal{K}$. Consequently, Corollary 2.4 can be applied to the model operator $A_{\Theta}$ in the case when the operator $A$ satisfies the following 
conditions: 1) $A$ is a contraction; 2) $\left\|A^{* n} x\right\| \rightarrow 0$ for all $x \in H$; 3) The defect operator $\mathcal{D}_{A^{*}}:=\left(I-A A^{*}\right)^{\frac{1}{2}}$ is compact.

In addition, assume that $\left\|A^{n} x\right\| \rightarrow 0$ for all $x \in H$. In this case, the subspace $E$ can be identified with $F$ and $\Theta(\xi)$ becomes unitary for almost all $\xi \in \mathbb{T}$. Consequently, Proposition 2.5 (shown below) is applicable to the model operator $A_{\Theta}$ in the case when the operator $A$ satisfies the following conditions: 1) $A$ is a contraction; 2) $\left\|A^{n} x\right\| \rightarrow 0$ and $\left\|A^{* n} x\right\| \rightarrow 0$ for all $x \in H$; 3 ) the defect operator $\mathcal{D}_{A^{*}}$ is compact.

Proposition 2.5. Let $A \in B(H)$ and assume that $I-A A^{*} \in K(H),\left\|A^{n} x\right\| \rightarrow 0$ and $\left\|A^{* n} x\right\| \rightarrow 0$ for all $x \in H$. For an arbitrary $T \in B(H)$, the following assertions are equivalent:

(a) The sequence $\left\{A^{* n} T A^{n}\right\}$ converges in operator norm.

(b) $A^{* n} T A^{n} \rightarrow 0$ in operator norm.

(c) $T$ is a compact operator.

Proof. (a) $\Rightarrow$ (b) By Corollary 2.4, $T=T_{0}+K$, where $A^{*} T_{0} A=T_{0}$ and $K \in K(H)$. On the other hand, by Lemma $2.3,\left\|A^{* n} K A^{n}\right\| \rightarrow 0$. It remains to show that $T_{0}=0$. Indeed, for an arbitrary $x, y \in H$, from the identity $A^{* n} T_{0} A^{n}=T_{0}(\forall n \in \mathbb{N})$, we can write

$$
\left|\left\langle T_{0} x, y\right\rangle\right|=\left|\left\langle T_{0} A^{n} x, A^{n} y\right\rangle\right| \leq\left\|T_{0}\right\|\left\|A^{n} x\right\|\left\|A^{n} y\right\| \rightarrow 0 .
$$

Hence $T_{0}=0$.

(b) $\Rightarrow(\mathrm{c}) \Rightarrow(\mathrm{a})$ are obtained from Lemma 2.3.

Recall that an operator $T \in B(X)$ is said to be almost periodic if for every $x \in X$, the orbit $\left\{T^{n} x: n \in \mathbb{N}\right\}$ is relatively compact. Clearly, an almost periodic operator is power bounded, that is,

$$
\sup _{n \geq 0}\left\|T^{n}\right\|<\infty .
$$

If $T \in B(X)$ is an almost periodic operator, then by the Jacobs-Glicksberg-de Leeuw decomposition theorem [7, Ch.I, Theorem 1.15], every $x \in X$ can be written as $x=x_{0}+x_{1}$, where $\left\|T^{n} x_{0}\right\| \rightarrow 0$ and $x_{1} \in \overline{\operatorname{span}}\{y \in X: \exists \xi \in \mathbb{T}, T y=\xi y\}$.

From now on, for a given $T \in B(X)$ the left and right multiplication operators on $B(X)$ will be denoted by $L_{T}$ and $R_{T}$, respectively.

The following result is an improvement of Proposition 2.5.

Proposition 2.6. Let $A \in B(H)$ and assume that $I-A A^{*} \in K(H),\left\|A^{n} x\right\| \rightarrow 0$ and $\left\|A^{* n} x\right\| \rightarrow 0$ for all $x \in H$. For an arbitrary $T \in B(H)$ the following assertions are equivalent:

(a) $\left\{A^{* n} T A^{n}: n \in \mathbb{N}\right\}$ is relatively compact in the operator norm topology.

(b) $A^{* n} T A^{n} \rightarrow 0$ in operator norm.

(c) $T$ is a compact operator.

Proof. (a) $\Rightarrow$ (b) Let $E$ be the set of all $Q \in B(H)$ such that

$$
\left\{\left(L_{A^{*}} R_{A}\right)^{n} Q: n \in \mathbb{N}\right\}
$$

is relatively compact in the operator norm topology. By the uniform boundedness principle, the operator $L_{A^{*}} R_{A}$ is power bounded and therefore $E$ is a closed 
(in operator norm) $L_{A^{*}} R_{A}$-invariant subspace. Consequently, $\left.L_{A^{*}} R_{A}\right|_{E}$, the restriction of $L_{A^{*}} R_{A}$ to $E$ is an almost periodic operator. Since $T \in E$, by the Jacobs-Glicksberg-de Leeuw decomposition theorem, $T=T_{0}+T_{1}$, where

$$
\lim _{n \rightarrow \infty}\left\|A^{* n} T_{0} A^{n}\right\|=0
$$

and

$$
T_{1} \in \overline{\operatorname{span}}^{\|\cdot\|}\left\{Q \in E: \exists \xi \in \mathbb{T}, A^{*} Q A=\xi Q\right\} .
$$

We must show that $T_{1}=0$. For this, it suffices to show that the identity $A^{*} Q A=\xi Q$ $(\xi \in \mathbb{T})$ implies $Q=0$. Indeed, since

$$
A^{* n} Q A^{n}=\xi^{n} Q, \forall n \in \mathbb{N},
$$

we get

$$
|\langle Q x, y\rangle|=\left|\left\langle Q A^{n} x, A^{n} y\right\rangle\right| \leq\|Q\|\left\|A^{n} x\right\|\left\|A^{n} y\right\| \rightarrow 0, \quad \forall x, y \in H .
$$

Hence $Q=0$.

(b) $\Rightarrow(\mathrm{c}) \Rightarrow(\mathrm{a})$ are obtained from Lemma 2.3 .

Next, we have the following:

Theorem 2.7. Let $A$ and $B^{*}$ be two essentially isometric contractions on $H$ and assume that $\left\|A^{n} x\right\| \rightarrow 0$ and $\left\|B^{* n} x\right\| \rightarrow 0$ for all $x \in H$. Then, for an arbitrary $T \in B(H)$ we have

$$
\lim _{n \rightarrow \infty}\left\|A^{n} T B^{n}\right\|=\|T+K(H)\| .
$$

Proof. If $K \in K(H)$, then by Lemma $2.3,\left\|A^{n} K B^{n}\right\| \rightarrow 0$. Since

$$
\left\|A^{n}(T+K) B^{n}\right\| \leq\|T+K\|,
$$

we have

$$
\lim _{n \rightarrow \infty}\left\|A^{n} T B^{n}\right\| \leq\|T+K(H)\| .
$$

For the reverse inequality, let $\widehat{A}, \widehat{T}$ and $\widehat{B}$ be the limit operators associated with $A, T$ and $B$, respectively. By Proposition $2.2, \widehat{A}$ and $\widehat{B}^{*}$ are isometries. By using the same proposition again, we can write

$$
\|T+K(H)\|=\|\widehat{T}\|=\left\|\widehat{A}^{n} \widehat{T} \widehat{B}^{n}\right\| \leq\left\|A^{n} T B^{n}\right\|, \quad \forall n \in \mathbb{N} .
$$

Thus we have

$$
\|T+K(H)\| \leq \lim _{n \rightarrow \infty}\left\|A^{n} T B^{n}\right\| .
$$

We know [5, Corollary 7.13] that every Toeplitz operator $T_{\varphi}$ with symbol $\varphi \in L^{\infty}$ satisfies

$$
\left\|T_{\varphi}\right\|=\left\|T_{\varphi}+K\left(H^{2}\right)\right\| .
$$

As a consequence of Theorem 2.7, we have the following generalization of the preceding formula.

Corollary 2.8. Let $A \in B(H)$ be a contraction and assume that $I-A A^{*} \in K(H)$ and $\left\|A^{* n} x\right\| \rightarrow 0$ for all $x \in H$. Then, for an arbitrary $T \in B(H)$ we have

$$
\lim _{n \rightarrow \infty}\left\|A^{* n} T A^{n}\right\|=\|T+K(H)\| \text {. }
$$


For an arbitrary $A, B \in B(H)$, we put

$$
\mathcal{I}_{A, B}=\{T \in B(H): A T B=T\} .
$$

Proposition 2.9. Assume that the operators $A, B$ satisfy the hypotheses of Theorem 2.7. Then, for an arbitrary $K \in K(H)$ we have

$$
\left\|K+\mathcal{I}_{A, B}\right\| \geq \frac{1}{2}\|K\| .
$$

In the case $A B=I$, this estimate is the best possible.

Proof. Assume that there exists $K \in K(H)$ such that

$$
\left\|K+\mathcal{I}_{A, B}\right\|<\frac{1}{2}\|K\| .
$$

Then there exists $T \in \mathcal{I}_{A, B}$ such that

$$
\|K+T\|<\frac{1}{2}\|K\|
$$

By Theorem 2.7, $\|T\|=\|T+K(H)\|$ which implies $\|T\| \leq\|K+T\|$. Consequently, we can write

$$
\|K\| \leq\|K+T\|+\|T\| \leq 2\|K+T\|<\|K\|,
$$

which is a contradiction.

In the case $A B=I$, we have $I \in \mathcal{I}_{A, B}$. If $K=x \otimes x$, where $\|x\|=1$ and $T=-\frac{1}{2} I$, then $\|K+T\|=\frac{1}{2}$.

Let $\mathcal{T}$ be the space of all Toeplitz operators. By taking $A=S^{*}$ and $B=S$ in Proposition 2.9, we have

$$
\|K+\mathcal{T}\| \geq \frac{1}{2}\|K\|, \quad \forall K \in K\left(H^{2}\right),
$$

where this estimate is the best possible.

\section{One dimensional model and the Hartman-Sarason theorem}

Using the results of the preceding section, here we give a quantitative generalization of the Hartman-Sarason theorem.

Recall that a contraction $T$ on $H$ is said to be completely non-unitary if it has no proper reducing subspace on which it acts as a unitary operator. If $T$ is a completely non-unitary contraction, then $f(T)\left(f \in H^{\infty}\right)$ can be defined by the Nagy-Foiaş functional calculus [19, Ch.III].

Let $T$ be a contraction on $H$ and assume that

$$
\lim _{n \rightarrow \infty}\left\|T^{n} x\right\|=\lim _{n \rightarrow \infty}\left\|T^{* n} x\right\|=0, \quad \forall x \in H .
$$

In addition, if

$$
\operatorname{dim}\left(I-T T^{*}\right) H=\operatorname{dim}\left(I-T^{*} T\right) H=1,
$$

then by the Model Theorem of Nagy-Foias [19, Ch.VI, Theorem 2.3] (see also, 21]) $T$ is unitary equivalent to its model operator

$$
S_{\theta}=\left.P_{\theta} S\right|_{H_{\theta}^{2}}
$$

acting on the model space

$$
H_{\theta}^{2}=H^{2} \ominus \theta H^{2}
$$


where $\theta$ is an inner function (a function $\theta$ in $H^{\infty}$ is an inner function if $|\theta|=1$ a.e. on $\mathbb{T}$ ) and $P_{\theta}$ is the orthogonal projection from $H^{2}$ onto $H_{\theta}^{2}$. Beurling's theorem [5. Corollary 6.11] says that these spaces are generic invariant subspaces for the backward shift operator

$$
\left(S^{*} f\right)(z)=\frac{f(z)-f(0)}{z}, \quad f \in H^{2} .
$$

Notice that

$$
S_{\theta}=\left(\left.S^{*}\right|_{H_{\theta}^{2}}\right)^{*}
$$

Let $\theta$ be an inner function and let $S_{\theta}$ be the model operator on the model space $H_{\theta}^{2}$. For an arbitrary $f \in H^{\infty}$, we can define the operator

$$
f\left(S_{\theta}\right)=\left.P_{\theta} f(S)\right|_{H_{\theta}^{2}}
$$

which is unitary equivalent to $f(T)$. The map $f \mapsto f\left(S_{\theta}\right)$ is linear, multiplicative and by the Nehari formula [21, p.235],

$$
\left\|f\left(S_{\theta}\right)\right\|=\operatorname{dist}\left(\bar{\theta} f, H^{\infty}\right) .
$$

Let us mention Sarason's theorem [21, p.230] which asserts that an operator $Q \in$ $B\left(H_{\theta}^{2}\right)$ is a commutant of $S_{\theta}$ if and only if $Q=f\left(S_{\theta}\right)$ for some $f \in H^{\infty}$.

Let us also mention that the classical theorem of Hartman and Sarason [21, p.235] classifies compactness of the operators $f\left(S_{\theta}\right)$. The operator $f\left(S_{\theta}\right)\left(f \in H^{\infty}\right)$ is compact if and only if $\bar{\theta} f \in H^{\infty}+C(\mathbb{T})$.

We have the following quantitative generalization of the Hartman-Sarason theorem.

Theorem 3.1. Let $\theta$ be an inner function and let $S_{\theta}$ be the model operator on the model space $H_{\theta}^{2}$. Then, for an arbitrary $f \in H^{\infty}$ we have

$$
\left\|f\left(S_{\theta}\right)+K\left(H_{\theta}^{2}\right)\right\|=\operatorname{dist}\left(\bar{\theta} f, H^{\infty}+C(\mathbb{T})\right) .
$$

For the proof, we need several lemmas.

Lemma 3.2. Let $\left\{E_{n}\right\}$ be an increasing sequence of closed subspaces of a Banach space $X$. Then, for an arbitrary $x \in X$ we have

$$
\lim _{n \rightarrow \infty} \operatorname{dist}\left(x, E_{n}\right)=\operatorname{dist}\left(x, \overline{\bigcup_{n=1}^{\infty} E_{n}}\right) .
$$

Proof. If $x \in X$, then the sequence $\left\{\operatorname{dist}\left(x, E_{n}\right)\right\}$ is decreasing. Let

$$
\alpha:=\lim _{n \rightarrow \infty} \operatorname{dist}\left(x, E_{n}\right)=\inf _{n} \operatorname{dist}\left(x, E_{n}\right) .
$$

Since

$$
E_{n} \subseteq \overline{\bigcup_{n=1}^{\infty} E_{n}}
$$

we have

$$
\operatorname{dist}\left(x, \overline{\bigcup_{n=1}^{\infty} E_{n}}\right) \leq \operatorname{dist}\left(x, E_{n}\right)
$$


which implies

$$
\operatorname{dist}\left(x, \overline{\bigcup_{n=1}^{\infty} E_{n}}\right) \leq \alpha .
$$

If

$$
\operatorname{dist}\left(x, \overline{\bigcup_{n=1}^{\infty} E_{n}}\right)<\alpha,
$$

then $\left\|x-x_{0}\right\|<\alpha$ for some $x_{0} \in \bigcup_{n=1}^{\infty} E_{n}$. Consequently, $x_{0} \in E_{n_{0}}$ for some $n_{0}$. Hence $\operatorname{dist}\left(x, E_{n_{0}}\right)<\alpha$. This contradicts $\operatorname{dist}\left(x, E_{n_{0}}\right) \geq \alpha$.

Lemma 3.3. For an arbitrary $\varphi \in L^{\infty}$ we have

$$
\lim _{n \rightarrow \infty} \operatorname{dist}\left(\varphi, \bar{z}^{n} H^{\infty}\right)=\operatorname{dist}\left(\varphi, H^{\infty}+C(\mathbb{T})\right) .
$$

Proof. We know [5, Proposition 6.36] that $H^{\infty}+C(\mathbb{T})$ is a uniformly closed subalgebra of $L^{\infty}$ generated by $\bar{z}$ and $H^{\infty}$. If $E_{n}:=\bar{z}^{n} H^{\infty}$, then $\left\{E_{n}\right\}$ is an increasing sequence of closed subspaces of $L^{\infty}$. Since

$$
H^{\infty}+C(\mathbb{T})=\overline{\operatorname{span}}_{L^{\infty}}\left\{\bar{z}^{n} H^{\infty}: n \geq 0\right\}
$$

and

$$
\bar{z}^{n} f_{1}+\bar{z}^{m} f_{2}=\left(z^{m} f_{1}+z^{n} f_{2}\right) \bar{z}^{n+m} \in \bar{z}^{n+m} H^{\infty} \quad\left(f_{1}, f_{2} \in H^{\infty}\right),
$$

we have

$$
\overline{\bigcup_{n=1}^{\infty} E_{n}}=H^{\infty}+C(\mathbb{T}) .
$$

Applying Lemma 3.2 to the subspaces $\left\{E_{n}\right\}$, we obtain our result.

Now, we can prove Theorem 3.1.

Proof of Theorem 3.1. As we have noted above, the model operator $S_{\theta}$ is an essentially unitary contraction. Moreover, $\left\|S_{\theta}^{n} h\right\| \rightarrow 0$ and $\left\|S_{\theta}^{* n} h\right\| \rightarrow 0$ for all $h \in H_{\theta}^{2}$. If $T \in B\left(H_{\theta}^{2}\right)$, then by Theorem 2.7,

$$
\lim _{n \rightarrow \infty}\left\|S_{\theta}^{* n} T S_{\theta}^{n}\right\|=\left\|T+K\left(H_{\theta}^{2}\right)\right\|
$$

which implies

$$
\lim _{n \rightarrow \infty}\left\|T S_{\theta}^{n}\right\| \geq\left\|T+K\left(H_{\theta}^{2}\right)\right\| .
$$

If $K \in K\left(H_{\theta}^{2}\right)$, then as $\left\|K S_{\theta}^{n}\right\| \rightarrow 0$ (see, the proof of Lemma 2.3) we get

$$
\lim _{n \rightarrow \infty}\left\|T S_{\theta}^{n}\right\|=\lim _{n \rightarrow \infty}\left\|(T+K) S_{\theta}^{n}\right\| \leq\|T+K\|, \quad \forall K \in K\left(H_{\theta}^{2}\right) .
$$

It follows that

$$
\lim _{n \rightarrow \infty}\left\|T S_{\theta}^{n}\right\| \leq\left\|T+K\left(H_{\theta}^{2}\right)\right\| .
$$

Thus we have

$$
\lim _{n \rightarrow \infty}\left\|T S_{\theta}^{n}\right\|=\left\|T+K\left(H_{\theta}^{2}\right)\right\|, \quad \forall T \in B\left(H_{\theta}^{2}\right) .
$$

In particular, taking $T=f\left(S_{\theta}\right)$ we obtain

$$
\lim _{n \rightarrow \infty}\left\|S_{\theta}^{n} f\left(S_{\theta}\right)\right\|=\left\|f\left(S_{\theta}\right)+K\left(H_{\theta}^{2}\right)\right\| .
$$


Further, by the Nehari formula we can write

$$
\left\|S_{\theta}^{n} f\left(S_{\theta}\right)\right\|=\operatorname{dist}\left(\bar{\theta} z^{n} f, H^{\infty}\right)=\operatorname{dist}\left(\bar{\theta} f, \bar{z}^{n} H^{\infty}\right) .
$$

On the other hand, by Lemma 3.3,

$$
\lim _{n \rightarrow \infty}\left\|S_{\theta}^{n} f\left(S_{\theta}\right)\right\|=\lim _{n \rightarrow \infty} \operatorname{dist}\left(\bar{\theta} f, \bar{z}^{n} H^{\infty}\right)=\operatorname{dist}\left(\bar{\theta} f, H^{\infty}+C(\mathbb{T})\right) .
$$

Now, taking into account (3.1), finally we obtain

$$
\left\|f\left(S_{\theta}\right)+K\left(H_{\theta}^{2}\right)\right\|=\operatorname{dist}\left(\bar{\theta} f, H^{\infty}+C(\mathbb{T})\right) .
$$

The proof is complete.

Below, we present some applications of Theorem 3.1.

Let $X$ be a Banach space. As usual, $\sigma(T)$ will denote the spectrum of the operator $T \in B(X)$. Given $T \in B(X)$, we let $A_{T}$ denote the closure in the uniform operator topology of all polynomials in $T$. Then, $A_{T}$ is a commutative unital Banach algebra. The Gelfand space of $A_{T}$ can be identified with $\sigma_{A_{T}}(T)$, the spectrum of $T$ with respect to the algebra $A_{T}$. Since $\sigma(T)$ is a (closed) subset of $\sigma_{A_{T}}(T)$, for every $\lambda \in \sigma(T)$, there is a multiplicative functional $\phi_{\lambda}$ on $A_{T}$ such that $\phi_{\lambda}(T)=\lambda$. By $\widehat{Q}$ we will denote the Gelfand transform of $Q \in A_{T}$. Instead of $\widehat{Q}\left(\phi_{\lambda}\right)\left(=\phi_{\lambda}(Q)\right)$, where $\lambda \in \sigma(T)$, we will use the notation $\widehat{Q}(\lambda)$. It follows from the Shilov Theorem [5. Theorem 2.54] that if $T$ is a contraction, then

$$
\sigma_{A_{T}}(T) \cap \mathbb{T}=\sigma(T) \cap \mathbb{T} .
$$

The following result was obtained in [17.

Theorem 3.4. If $T$ is a contraction on a Hilbert space, then for an arbitrary $Q \in A_{T}$ we have

$$
\lim _{n \rightarrow \infty}\left\|T^{n} Q\right\|=\sup _{\xi \in \sigma(T) \cap \mathbb{T}}|\widehat{Q}(\xi)| .
$$

For a non-empty closed subset $\Gamma$ of $\mathbb{T}$, by $H_{\Gamma}^{\infty}$ we will denote the set of all those functions $f$ in $H^{\infty}$ that have a continuous extension $\widetilde{f}$ to $\mathbb{D} \cup \Gamma$. Clearly, $H_{\Gamma}^{\infty}$ is a closed subspace of $H^{\infty}$. It follows from the general theory of $H^{p}$ spaces that if $\Gamma$ has positive Lebesgue measure and $f \in H_{\Gamma}^{\infty}$ is not identically zero, then $\widetilde{f}$ cannot vanish identically on $\Gamma$.

If $T$ is a contraction on a Hilbert space $H$, then there is a canonical decomposition of $H$ into two $T$-reducing subspaces $H=H_{0} \oplus H_{u}$ such that $T_{0}:=\left.T\right|_{H_{0}}$ is completely non-unitary and $T_{u}:=\left.T\right|_{H_{u}}$ is unitary [19, Ch.I, Theorem 3.2]. It can be seen that

$$
\sigma\left(T_{u}\right) \subseteq \sigma(T) \cap \mathbb{T} .
$$

Let $f$ be in $H_{\sigma(T) \cap \mathbb{T}}^{\infty}$ with continuous extension $\widetilde{f}$ to $\mathbb{D} \cup(\sigma(T) \cap \mathbb{T})$. As in [10, we can define $f(T) \in B(H)$ by

$$
f(T)=f\left(T_{0}\right) \oplus \widetilde{f}\left(T_{u}\right),
$$

where $f\left(T_{0}\right)$ is given by the Nagy-Foias functional calculus and

$$
\tilde{f}\left(T_{u}\right)=\left(\left.\tilde{f}\right|_{\sigma(T) \cap \mathbb{T}}\right)\left(T_{u}\right) .
$$

It can be seen that

$$
\|f(T)\| \leq\|f\|_{\infty}, \quad \forall f \in H_{\sigma(T) \cap \mathbb{T}}^{\infty} .
$$


Further, by the Gamelin-Garnett theorem [9], there exists a sequence $\left\{f_{n}\right\}$ in $H^{\infty}$ such that each $f_{n}$ has an analytic extension $g_{n}$ to a neighborhood $O_{n}$ of $\mathbb{D} \cup(\sigma(T) \cap$ $\mathbb{T})$ and

$$
\lim _{n \rightarrow \infty}\left\|f_{n}-f\right\|_{\infty}=0 .
$$

Then, $g_{n}(T)$ can be defined by the Riesz-Dunford functional calculus. Since $f_{n}(T)=$ $g_{n}(T) \in A_{T}$ and

$$
\left\|f_{n}(T)-f(T)\right\| \leq\left\|f_{n}-f\right\|_{\infty} \rightarrow 0,
$$

we have that $f(T) \in A_{T}$. Moreover,

$$
\widehat{f(T)}(\xi)=\widetilde{f}(\xi), \quad \forall \xi \in \sigma(T) \cap \mathbb{T} .
$$

As a consequence of Theorem 3.4 we have the following:

Corollary 3.5. Let $T$ be a contraction on a Hilbert space. If $f \in H_{\sigma(T) \cap \mathbb{T}}^{\infty}$ with continuous extension $\tilde{f}$ to $\mathbb{D} \cup(\sigma(T) \cap \mathbb{T})$, then

$$
\lim _{n \rightarrow \infty}\left\|T^{n} f(T)\right\|=\sup _{\xi \in \sigma(T) \cap \mathbb{T}}|\tilde{f}(\xi)| .
$$

Now, let $\theta$ be an inner function and let $S_{\theta}$ be the model operator on the model space $H_{\theta}^{2}$. We put

$$
\Sigma_{u}(\theta)=\left\{\xi \in \mathbb{T}: \liminf _{z \in \mathbb{D}, z \rightarrow \xi}|\theta(z)|=0\right\}
$$

It follows from the Lipschitz-Moeller theorem [21, p.81] that

$$
\sigma\left(S_{\theta}\right) \cap \mathbb{T}=\Sigma_{u}(\theta) .
$$

If $f \in H_{\Sigma_{u}(\theta)}^{\infty}$ with continuous extension $\widetilde{f}$ to $\mathbb{D} \cup \Sigma_{u}(\theta)$, then by Corollary 3.5,

$$
\lim _{n \rightarrow \infty}\left\|S_{\theta}^{n} f\left(S_{\theta}\right)\right\|=\sup _{\xi \in \Sigma_{u}(\theta)}|\widetilde{f}(\xi)|
$$

On the other hand, by (3.1),

$$
\lim _{n \rightarrow \infty}\left\|S_{\theta}^{n} f\left(S_{\theta}\right)\right\|=\left\|f\left(S_{\theta}\right)+K\left(H_{\theta}^{2}\right)\right\| .
$$

Thus we have

$$
\left\|f\left(S_{\theta}\right)+K\left(H_{\theta}^{2}\right)\right\|=\sup _{\xi \in \Sigma_{u}(\theta)}|\widetilde{f}(\xi)| .
$$

From Theorem 3.1 and from the preceding identity we have the following:

Corollary 3.6. Let $\theta$ be an inner function and let $S_{\theta}$ be the model operator on the model space $H_{\theta}^{2}$. For an arbitrary $f \in H_{\Sigma_{u}(\theta)}^{\infty}$ with continuous extension $\tilde{f}$ to $\mathbb{D} \cup \Sigma_{u}(\theta)$, we have

$$
\left\|f\left(S_{\theta}\right)+K\left(H_{\theta}^{2}\right)\right\|=\operatorname{dist}\left(\bar{\theta} f, H^{\infty}+C(\mathbb{T})\right)=\sup _{\xi \in \Sigma_{u}(\theta)}|\widetilde{f}(\xi)|
$$




\section{The SEQUenCE $\left\{\frac{1}{n} \sum_{i=0}^{n-1} A^{i} T B^{i}\right\}$}

In this section, we give some results concerning convergence in operator norm of the sequence $\left\{\frac{1}{n} \sum_{i=0}^{n-1} A^{i} T B^{i}\right\}$ for Hilbert space operators.

Let $X$ be a Banach space. It is easy to check that if $T \in B(X)$ is power bounded, then

$$
\overline{(T-I) X}=\left\{x \in X: \lim _{n \rightarrow \infty}\left\|\frac{1}{n} \sum_{i=0}^{n-1} T^{i} x\right\|=0\right\} .
$$

The following result is well known (for instance, see [12, Ch.2, §2.1, Theorems 1.2 and 1.3]).

Proposition 4.1. Let $T \in B(X)$ be power bounded and let $E$ be the set of all $x \in X$ such that the sequence $\left\{\frac{1}{n} \sum_{i=0}^{n-1} T^{i} x\right\}$ converges strongly. Then, we have the decomposition

$$
E=\overline{(T-I) X} \oplus \operatorname{ker}(T-I)
$$

If $X$ is reflexive, then $E=X$.

Applying Proposition 4.1 to the operator $L_{A} R_{B}$ on the space $B(X)$, we have the following:

Corollary 4.2. Let $A, B \in B(X)$ be two operators such that $\sup _{n \geq 0}\left(\left\|A^{n}\right\|\left\|B^{n}\right\|\right)<$ $\infty$ and $T \in B(X)$. Then, the sequence $\left\{\frac{1}{n} \sum_{i=0}^{n-1} A^{i} T B^{i}\right\}$ converges in operator norm if and only if we have the decomposition $T=T_{0}+Q$, where

$$
\left\|\frac{1}{n} \sum_{i=0}^{n-1} A^{i} T_{0} B^{i}\right\| \rightarrow 0 \text { and } A Q B=Q .
$$

Lemma 4.3. Let $T \in B(X)$ be power bounded, $x \in X$ and assume that

$$
\lim _{n \rightarrow \infty}\left\|T^{n+1} x-T^{n} x\right\|=0 .
$$

(a) If the sequence $\left\{\frac{1}{n} \sum_{i=0}^{n-1} T^{i} x\right\}$ converges strongly, then the sequence $\left\{T^{n} x\right\}$ converges strongly (to same element), too.

(b) If $X$ is reflexive, then the sequence $\left\{T^{n} x\right\}$ converges strongly.

Proof. (a) Notice that

$$
F:=\left\{y \in X: \lim _{n \rightarrow \infty}\left\|T^{n+1} y-T^{n} y\right\|=0\right\}
$$

is a closed $T$-invariant subspace and $x \in F$. Since $T$ is power bounded and

$$
\left\|T^{n}(T-I) y\right\|=\left\|T^{n+1} y-T^{n} y\right\| \rightarrow 0, \forall y \in F
$$

we have $\left\|T^{n} y\right\| \rightarrow 0$ for all $y \in \overline{(T-I) F}$. Now, let $E$ be the set of all $y \in F$ such that the sequence $\left\{\frac{1}{n} \sum_{i=0}^{n-1} T^{i} y\right\}$ converges strongly. Since $x \in E$, by Proposition 4.1 we have the decomposition $x=x_{0}+y_{0}$, where $x_{0} \in \overline{(T-I) F}$ and $T y_{0}=$ $y_{0}$. As $T^{n} x=T^{n} x_{0}+y_{0}$ and $\left\|T^{n} x_{0}\right\| \rightarrow 0$, we have $\left\|T^{n} x-y_{0}\right\| \rightarrow 0$. Clearly, $\frac{1}{n} \sum_{i=0}^{n-1} T^{i} x \rightarrow y_{0}$ strongly.

(b) If $X$ is reflexive, then by Proposition 4.1 the sequence $\left\{\frac{1}{n} \sum_{i=0}^{n-1} T^{i} x\right\}$ converges strongly for every $x \in X$. By (a), the sequence $\left\{T^{n} x\right\}$ converges strongly. 
Next, we have the following:

Theorem 4.4. Let $A$ and $B^{*}$ be two essentially isometric operators on $H$ and $T \in B(H)$. Assume that:

(i) $\left\|A^{n} x\right\| \rightarrow 0$ and $\left\|B^{* n} x\right\| \rightarrow 0$ for all $x \in H$;

(ii) $A T B-T \in K(H)$.

Then, the sequence $\left\{\frac{1}{n} \sum_{i=0}^{n-1} A^{i} T B^{i}\right\}$ converges in operator norm if and only if we have the decomposition $T=T_{0}+K$, where $A T_{0} B=T_{0}$ and $K \in K(H)$.

Proof. Assume that the sequence $\left\{\frac{1}{n} \sum_{i=0}^{n-1} A^{i} T B^{i}\right\}$ converges in operator norm. Since $A T B-T \in K(H)$, by Lemma 2.3,

$$
\lim _{n \rightarrow \infty}\left\|\left(L_{A} R_{B}\right)^{n+1} T-\left(L_{A} R_{B}\right)^{n} T\right\|=\lim _{n \rightarrow \infty}\left\|A^{n}(A T B-T) B^{n}\right\|=0 .
$$

Notice also that the operator $L_{A} R_{B}$ is power bounded. Applying Lemma 4.3 to the operator $L_{A} R_{B}$ on the space $B(X)$, we obtain that the sequence $\left\{A^{n} T B^{n}\right\}$ converges in operator norm. By Theorem 2.1, $T=T_{0}+K$, where $A T_{0} B=T_{0}$ and $K \in K(H)$.

If $T=T_{0}+K$, where $A T_{0} B=T_{0}$ and $K \in K(H)$, then we have

$$
\frac{1}{n} \sum_{i=0}^{n-1} A^{i} T B^{i}=T_{0}+\frac{1}{n} \sum_{i=0}^{n-1} A^{i} K B^{i} .
$$

By Lemma 2.3, $\left\|A^{n} K B^{n}\right\| \rightarrow 0$ and therefore $\left\|\frac{1}{n} \sum_{i=0}^{n-1} A^{i} K B^{i}\right\| \rightarrow 0$. Thus

$$
\frac{1}{n} \sum_{i=0}^{n-1} A^{i} T B^{i} \rightarrow T_{0} \text { in operator norm. }
$$

Corollary 4.5. Assume that the operators $A, T \in B(H)$ satisfy the following conditions:

(i) $I-A A^{*} \in K(H)$;

(ii) $\left\|A^{* n} x\right\| \rightarrow 0$ for all $x \in H$;

(iii) $A^{*} T A-T \in K(H)$.

Then, the sequence $\left\{\frac{1}{n} \sum_{i=0}^{n-1} A^{* i} T A^{i}\right\}$ converges in operator norm if and only if we have the decomposition $T=T_{0}+K$, where $A^{*} T_{0} A=T_{0}$ and $K \in K(H)$.

The proof of the following lemma is straightforward and will be omitted.

Lemma 4.6. Let $U$ be an essentially unitary operator on $H$. Then, $T \in B(H)$ is an essential commutant of $U$ if and only if $U^{*} T U-T \in K(H)$.

Recall that $T \in B\left(H^{2}\right)$ is an essentially Toeplitz operator if

$$
S^{*} T S-T \in K\left(H^{2}\right) .
$$

By Lemma 4.6, $T \in B\left(H^{2}\right)$ is an essentially Toeplitz operator if and only if $T$ is an essential commutant of the unilateral shift operator $S$. On the other hand, essential commutant of the unilateral shift is a $C^{*}$-algebra. Consequently, the set of all essentially Toeplitz operators is a $C^{*}$-algebra and therefore contains the $C^{*}$-algebra generated by all Toeplitz operators. 
Corollary 4.7. An essentially Toeplitz operator $T$ is a compact perturbation of a Toeplitz operator if and only if the sequence $\left\{\frac{1}{n} \sum_{i=0}^{n-1} S^{* i} T S^{i}\right\}$ converges in operator norm.

In 20, it was proved that if the composition operator $C_{\phi}$ on $H^{2}$ is neither compact nor the identity, then $C_{\phi}$ cannot be compact perturbation of a Toeplitz operator.

Corollary 4.8. If $C_{\phi}$ is a composition operator on $H^{2}$, then the sequence

$$
\left\{\frac{1}{n} \sum_{i=0}^{n-1} S^{* i} C_{\phi} S^{i}\right\}
$$

converges in operator norm if and only if either $C_{\phi}$ is compact or the identity operator.

Recall that the class of compact composition operators are sufficiently large (for instance, see [16]).

Following [15, we could define an asymptotic Toeplitz operator in the Calkin algebra as an operator $T \in B\left(H^{2}\right)$ such that the sequence $\left\{S^{* n} T S^{n}\right\}$ converges in the Calkin algebra.

The following result, which seems to be unnoticed (see, [15, p.745]).

Proposition 4.9. Every asymptotic Toeplitz operator in the Calkin algebra is an essentially Toeplitz operator.

Proof. If $T \in B\left(H^{2}\right)$ is an asymptotic Toeplitz operator in the Calkin algebra, then there is an operator $Q \in B\left(H^{2}\right)$ such that

$$
\lim _{n \rightarrow \infty}\left\|S^{* n} T S^{n}-Q+K(H)\right\|=0 .
$$

Let $\widehat{S^{*}}, \widehat{T}, \widehat{S}$ and $\widehat{Q}$ be the limit operators associated with $S^{*}, T, S$ and $Q$, respectively. By Proposition 2.2,

$$
\lim _{n \rightarrow \infty}\left\|\widehat{S}^{n} \widehat{T}^{n}-\widehat{Q}\right\|=0
$$

Since

$$
\lim _{n \rightarrow \infty}\left\|\widehat{S}^{n+1} \widehat{T} \widehat{S}^{n+1}-\widehat{S^{*}} \widehat{Q} \widehat{S}\right\|=0,
$$

we have $\widehat{S^{*}} \widehat{Q} \widehat{S}=\widehat{Q}$. By using the same proposition again, we obtain that $S^{*} Q S-$ $Q \in K\left(H^{2}\right)$.

\section{BANACH SPACE OPERATORS}

In this section, we study convergence in operator norm of the sequence $\left\{A^{n} T B^{n}\right\}$ for Banach space operators.

Let $X$ be a Banach space. For an arbitrary $T \in B(X)$ and $x \in X$, we define $\rho_{T}(x)$ to be the set of all $\lambda \in \mathbb{C}$ for which there exists a neighborhood $U_{\lambda}$ of $\lambda$ with $u(z)$ analytic on $U_{\lambda}$ having values in $X$ such that

$$
(z I-T) u(z)=x, \forall z \in U_{\lambda} .
$$

This set is open and contains the resolvent set $\rho(T)$ of $T$. By definition, the local spectrum of $T$ at $x \in X$, denoted by $\sigma_{T}(x)$, is the complement of $\rho_{T}(x)$, so it is a compact subset of $\sigma(T)$. This object is the most tractable if the operator $T$ has the 
single-valued extension property (SVEP), i.e., for every open set $U$ in $\mathbb{C}$, the only analytic function $u: U \rightarrow X$ for which the equation $(z I-T) u(z)=0$ holds is the constant function $u \equiv 0$. If $T$ has SVEP, then $\sigma_{T}(x) \neq \emptyset$, whenever $x \in X \backslash\{0\}$ [13, Proposition 1.2.16]. Note that the local spectrum of $T$ may be "very small" with respect to its usual spectrum. To see this, let $\sigma$ be a "small" clopen part of $\sigma(T)$. Let $P_{\sigma}$ be the spectral projection associated with $\sigma$ and $X_{\sigma}:=P_{\sigma} X$. Then, $X_{\sigma}$ is a closed $T$-invariant subspace of $X$ and $\sigma\left(\left.T\right|_{X_{\sigma}}\right)=\sigma$. It is easy to see that $\sigma_{T}(x) \subseteq \sigma$ for every $x \in X_{\sigma}$.

If $T$ is power bounded, then clearly, $\sigma(T) \subset \overline{\mathbb{D}}$ and $\sigma_{T}(x) \cap \mathbb{T}$ consists of all $\xi \in \mathbb{T}$ such that the function $z \rightarrow(z I-T)^{-1} x(|z|>1)$ has no analytic extension to a neighborhood of $\xi$.

Lemma 5.1. Let $T \in B(X), x \in X$ and assume that $\sup _{n \geq 0}\left\|T^{n} x\right\|<\infty$. Then, $\sigma_{T}(x) \subseteq \overline{\mathbb{D}}$.

Proof. Consider the function

$$
u(z):=\sum_{n=0}^{\infty} \frac{T^{n} x}{z^{n+1}}
$$

which is analytic on $\mathbb{C} \backslash \overline{\mathbb{D}}$ and $(z I-T) u(z)=x$ for all $z \in \mathbb{C} \backslash \overline{\mathbb{D}}$. This shows that $\mathbb{C} \backslash \overline{\mathbb{D}} \subseteq \rho_{T}(x)$ and therefore $\sigma_{T}(x) \subseteq \overline{\mathbb{D}}$.

We mention the following classical result of Katznelson and Tzafriri 11, Theorem 1]: If $T \in B(X)$ is power bounded, then $\lim _{n \rightarrow \infty}\left\|T^{n+1}-T^{n}\right\|=0$ if and only if $\sigma(T) \cap \mathbb{T} \subseteq\{1\}$.

We have the following local version of the Katznelson-Tzafriri theorem [18, Theorem 4.2$]$.

Theorem 5.2. Let $T \in B(X), x \in X$ and assume that $\sup _{n \geq 0}\left\|T^{n} x\right\|<\infty$. If $\sigma_{T}(x) \cap \mathbb{T} \subseteq\{1\}$, then

$$
\lim _{n \rightarrow \infty}\left\|T^{n+1} x-T^{n} x\right\|=0 .
$$

Note that in contrast with the Katznelson-Tzafriri theorem, the converse of Theorem 5.2 does not hold, in general. Indeed, if $S^{*}$ is the backward shift operator on $H^{2}$, then as $\left\|S^{* n} f\right\| \rightarrow 0$, we have

$$
\lim _{n \rightarrow \infty}\left\|S^{*(n+1)} f-S^{* n} f\right\|=0, \quad \forall f \in H^{2} .
$$

On the other hand, since

$$
\left(\lambda I-S^{*}\right)^{-1} f(z)=\frac{\lambda^{-1} f\left(\lambda^{-1}\right)-z f(z)}{1-\lambda z} \quad(|\lambda|>1),
$$

$\sigma_{S^{*}}(f) \cap \mathbb{T}$ consists of all $\xi \in \mathbb{T}$ for which the function $f$ has no analytic extension to a neighborhood of $\xi$ (see, [6, p.24]).

Theorem 5.2 combined with Lemma 4.3 yields the next result.

Theorem 5.3. Assume that $T \in B(X)$ and $x \in X$ satisfy the following conditions:

(i) $\sup _{n \geq 0}\left\|T^{n} x\right\|<\infty$;

(ii) $\sigma_{T}(x) \cap \mathbb{T} \subseteq\{1\}$.

If the sequence $\left\{\frac{1}{n} \sum_{i=0}^{n-1} T^{i} x\right\}$ converges strongly to $y \in X$, then $T^{n} x \rightarrow y$ strongly. 
Corollary 5.4. Let $T \in B(X)$ and let $x \in X$ be such that $\sup _{n \geq 0}\left\|T^{n} x\right\|<\infty$. Let

$$
S:=\frac{I+T+\ldots+T^{k-1}}{k}(k>1 \text { is a fixed integer })
$$

and assume that the sequence $\left\{\frac{1}{n} \sum_{i=0}^{n-1} S^{i} x\right\}$ converges strongly to $y \in X$. Then, $S^{n} x \rightarrow y$ strongly.

Proof. It is easy to check that

$$
\sup _{n \geq 0}\left\|S^{n} x\right\| \leq \sup _{n \geq 0}\left\|T^{n} x\right\|<\infty .
$$

Notice also that if

$$
f(z):=\frac{1+z+\ldots+z^{k-1}}{k}(z \in \mathbb{C})
$$

then $f(1)=1$ and $|f(z)|<1$ for all $z \in \overline{\mathbb{D}} \backslash\{1\}$. On the other hand, by [13, Theorem 3.3.8],

$$
\sigma_{S}(x)=\sigma_{f(T)}(x)=f\left(\sigma_{T}(x)\right) .
$$

Since $\sigma_{T}(x) \subseteq \overline{\mathbb{D}}$ (Lemma 5.1), we have $\sigma_{S}(x) \cap \mathbb{T} \subseteq\{1\}$. By Theorem 5.3, $S^{n} x \rightarrow y$ strongly.

We put

$$
D_{+}=\{z \in \mathbb{C}: \operatorname{Re} z \geq 1, \operatorname{Im} z \geq 0\} \text { and } D_{-}=\{z \in \mathbb{C}: \operatorname{Re} z \geq 1, \operatorname{Im} z \leq 0\} .
$$

As another application of Theorem 5.3, we have the following:

Theorem 5.5. Assume that the operators $A, T, B \in B(X)$ satisfy the following conditions:

(i) $\sup _{n \geq 0}\left\|A^{n} T B^{n}\right\|<\infty$;

(ii) either $\sigma(A) \subset D_{+}$and $\sigma(B) \subset D_{-}$or $\sigma(A) \subset D_{-}$and $\sigma(B) \subset D_{+}$.

If the sequence $\left\{\frac{1}{n} \sum_{i=0}^{n-1} A^{i} T B^{i}\right\}$ converges in operator norm to $Q \in B(X)$, then $A^{n} T B^{n} \rightarrow Q$ in operator norm.

Proof. Since

$$
\sup _{n \geq 0}\left\|\left(L_{A} R_{B}\right)^{n} T\right\|=\sup _{n \geq 0}\left\|A^{n} T B^{n}\right\|<\infty,
$$

by Lemma 5.1,

$$
\sigma_{L_{A} R_{B}}(T) \subseteq \overline{\mathbb{D}}
$$

On the other hand, by the Lumer-Rosenblum theorem [14, Theorem 10],

$$
\sigma\left(L_{A} R_{B}\right)=\{\lambda \mu: \lambda \in \sigma(A), \mu \in \sigma(B)\}
$$

which implies

$$
\sigma_{L_{A} R_{B}}(T) \subseteq \sigma\left(L_{A} R_{B}\right) \subset\{z \in \mathbb{C}: \operatorname{Re} z \geq 1\} .
$$

Thus we have

$$
\sigma_{L_{A} R_{B}}(T) \subseteq \overline{\mathbb{D}} \cap\{z \in \mathbb{C}: \operatorname{Re} z \geq 1\}=\{1\} .
$$

Applying Theorem 5.3 to the operator $L_{A} R_{B}$ on the space $B(X)$, we obtain that $A^{n} T B^{n}=\left(L_{A} R_{B}\right)^{n} T \rightarrow Q$ in operator norm. 
Next, we will show that the hypothesis $\sigma_{T}(x) \cap \mathbb{T} \subseteq\{1\}$ in Theorem 5.3 is the best possible, in general.

Let $N$ be a normal operator on a Hilbert space $H$ with the spectral measure $P$ and $x \in H$. Define a measure $\mu_{x}$ on $\sigma(N)$ by

$$
\mu_{x}(\Delta)=\langle P(\Delta) x, x\rangle=\|P(\Delta) x\|^{2} .
$$

It follows from the Spectral Theorem that $\sigma(N)=\operatorname{supp} P$ and $\sigma_{N}(x)=\operatorname{supp} \mu_{x}$. It is easy to check that if $N$ is a contraction (a normal operator is power bounded if and only if it is a contraction) then,

$$
\frac{1}{n} \sum_{i=0}^{n-1} N^{i} x \rightarrow P(\{1\}) x \text { in norm for all } x \in H .
$$

Proposition 5.6. Let $N$ be a normal contraction operator on $H$ with the spectral measure $P$ and $x \in H$. The sequence $\left\{N^{n} x\right\}$ converges strongly if and only if

$$
P\left(\sigma_{N}(x) \cap \mathbb{T} \backslash\{1\}\right) x=0 .
$$

In this case, $N^{n} x \rightarrow P(\{1\}) x$ strongly.

Proof. Let $\mu_{x}$ be the measure on $\sigma(N)$ defined by (5.1). We can write

$$
\begin{aligned}
& \lim _{n \rightarrow \infty}\left\|N^{n+1} x-N^{n} x\right\|^{2}=\lim _{n \rightarrow \infty} \int_{\sigma_{N}(x)}\left|z^{n+1}-z^{n}\right|^{2} d \mu_{x}(z) \\
= & \lim _{n \rightarrow \infty} \int_{\sigma_{N}(x) \backslash\left(\sigma_{N}(x) \cap \mathbb{T}\right)}|z|^{2 n}|z-1|^{2} d \mu_{x}(z) \\
& +\lim _{n \rightarrow \infty} \int_{\sigma_{N}(x) \cap \mathbb{T}}|z|^{2 n}|z-1|^{2} d \mu_{x}(z) \\
= & \int_{\sigma_{N}(x) \cap \mathbb{T}}|z-1|^{2} d \mu_{x}(z)=\int_{\sigma_{N}(x) \cap \mathbb{T} \backslash\{1\}}|z-1|^{2} d \mu_{x}(z) .
\end{aligned}
$$

It follows that $\left\|N^{n+1} x-N^{n} x\right\| \rightarrow 0$ if and only if

$$
\mu_{x}\left(\sigma_{N}(x) \cap \mathbb{T} \backslash\{1\}\right)=0 .
$$

By Lemma 4.3 the sequence $\left\{N^{n} x\right\}$ converges strongly if and only if

$$
P\left(\sigma_{N}(x) \cap \mathbb{T} \backslash\{1\}\right) x=0 .
$$

By (5.2),

$$
\lim _{n \rightarrow \infty} N^{n} x=\lim _{n \rightarrow \infty} \frac{1}{n} \sum_{i=0}^{n-1} N^{i} x=P(\{1\}) x .
$$

Let $W^{*}(N)$ be the von Neumann algebra generated by $N$. Recall that $x \in H$ is a separating vector for $N$ if the only operator $A$ in $W^{*}(N)$ such that $A x=0$ is $A=0$. As is known [4, Ch.IX, Section 8.1], each normal operator has a separating vector. If $x \in H$ is a separating vector for $N$, then the spectral measure of $N$ and the measure $\mu_{x}$ are mutually absolutely continuous [4, Ch.IX, Proposition 8.3], where $\mu_{x}$ is defined by (5.1). 
Corollary 5.7. If $x$ is a separating vector for $N$, then the sequence $\left\{N^{n} x\right\}$ converges strongly if and only if

$$
P\left(\sigma_{N}(x) \cap \mathbb{T} \backslash\{1\}\right)=0 .
$$

Now, let $K$ be a compact subset of $\overline{\mathbb{D}}$ such that $1 \in K$ and let $\nu$ be a regular positive Borel measure in $\mathbb{C}$ with support $K$. Define the operator $N$ on $L^{2}(K, \nu)$ by $N f=z f$. Then, $N$ is a normal contraction on $L^{2}(K, \nu)$ and $\sigma(N)=K$. Moreover,

$$
P(\Delta) f=\chi_{\Delta} f, \quad \forall f \in L^{2}(K, \nu),
$$

where $\chi_{\Delta}$ is the characteristic function of $\Delta$. It can be seen that the identity one function $\mathbf{1}$ on $K$ is a separating vector for $N$ and $\sigma(N)=\sigma_{N}(\mathbf{1})$. By (5.3), the sequence $\left\{N^{n} \mathbf{1}\right\}$ converges strongly if and only if $\chi_{\sigma_{N}(\mathbf{1}) \cap \mathbb{T}}=\chi_{\{1\}}$ or $\sigma_{N}(\mathbf{1}) \cap \mathbb{T}=$ $\{1\}$.

\section{REFERENCES}

[1] J. Barria and P. R. Halmos, Asymptotic Toeplitz operators, Trans. Amer. Math. Soc. 273(1982), 621-630.

[2] B. Beauzamy, Introduction to Operator Theory and Invariant Subspaces, North Holland, Amsterdam, 1988.

[3] A. Brown and P. R. Halmos, Algebraic properties of Toeplitz operators, J. Reine Angew. Math. 213(1963/1964), 89-102.

[4] J. B. Conway, A Course in Functional Analysis, Grad. Texts in Math. Springer-Verlag, 1985.

[5] R. G. Douglas, Banach Algebra Techniques in Operator Theory, Academic Press, New York, 1972.

[6] N. Dunford and J. T. Schwartz, Linear Operators III (Russian), Mir, Moscow, 1974.

[7] T. Eisner, Stability of Operators and Operator Semigroups, Oper. Theory Adv. Appl., Birkhäuser, Basel, Vol. 209, 2010.

[8] A. Feintuch, On asymptotic Toeplitz and Hankel operators, In the Gohberg anniversary collection, Oper. Theory Adv. Appl. 41(1989), 241-254.

[9] T. Gamelin and J. Garnett, Uniform approximation to bounded analytic functions, Rev. Un. Math. Argentina, 25(1970), 87-94.

[10] I.B. Jung, E. Ko and C. Pearcy, A note on the spectral mapping theorem, Kyungpook Math. J. 47(2007), 77-79.

[11] Y. Katznelson and L. Tzafriri, On power bounded operators, J. Funct. Anal. 68(1986), 313328.

[12] U. Krengel, Ergodic Theorems, Walter de Gruyter, Berlin, New York, 1985.

[13] K.B. Laursen and M.M. Neumann, An Introduction to Local Spectral Theory, Oxford, Clarendon Press, 2000.

[14] G. Lumer and M. Rosenblum, Linear operator equations, Proc. Amer. Math. Soc. 10(1959), $32-41$.

[15] R. A. Martinez-Avendaño, Essentially Hankel operators, J. London Math. Soc. 66(2002), 741-752.

[16] R. A. Martinez-Avendaño and P. Rosenthal, An Introduction to Operators on the HardyHilbert Space, Grad. Texts in Math. 237, Springer, 2007.

[17] H. S. Mustafayev, Asymptotic behavior of polynomially bounded operators, C. R. Acad. Sci. Paris, Ser. I. 348(2010), 517-520.

[18] H. S. Mustafayev, Growth conditions for conjugate orbits of operators on Banach spaces, J. Oper. Theory, 74(2015), 281-306.

[19] B. Sz.-Nagy and C. Foias, Harmonic Analysis of Operators on Hilbert Space (Russian), Mir, Moscow, 1970.

[20] F. Nazarov and J.H. Shapiro, On the toeplitzness of composition operators, Complex Var. Elliptic Equ. 52(2007), 193-210.

[21] N. K. Nikolski, Treatise on the Shift Operator (Russian), Nauka, Moscow, 1980. 
Institute of Mathematics and Mechanics of Academy Sciences of Azerbaijan, BaKUAZERBAIJAN

E-mail address: hsmustafayev@yahoo.com 